\title{
Gastric mucosal density of Helicobacter pylori estimated by real-time PCR compared with results of urea breath test and histological grading
}

\author{
D. KOBAYASHI, Y. EISHI, T. OHKUSA*, I. ISHIGE, T. SUZUKI, J. MINAMI, T. YAMADA, \\ T. TAKIZAWA and M. KOIKE \\ Department of Human Pathology, School of Medicine, Tokyo Medical and Dental University, Tokyo and \\ * Department of Gastroenterology, Juntendo University, School of Medicine, Tokyo, Japan
}

\begin{abstract}
The accuracy of the urea breath test (UBT) and histological grading for estimation of the density of Helicobacter pylori in gastric mucosa is not known. Real-time (TaqMan) PCR was used to estimate the total number of $\mathrm{H}$. pylori genomes in biopsy samples. These values were compared with those obtained by the UBT and the histological grade obtained by the Sydney system. The UBT and endoscopy with antral and corporal biopsies were performed in 88 consecutive untreated patients with dyspepsia. Bacterial culture and the rapid urease test were done with fresh biopsy materials. TaqMan PCR and histological examination were done on serial paraffin sections of the biopsy samples. Of the five methods tested, TaqMan PCR had the highest sensitivity and specificity (both $100 \%$ ) in the diagnosis of $\mathrm{H}$. pylori infection. The mean density of $\mathrm{H}$. pylori genomes for pairs of biopsy samples from individual patients was compared with the individual values obtained by the UBT; correlation between the results was significant. The density of $H$. pylori genomes was higher in histological grades 1,2 and 3 than in grade 0 , without significant differences between adjacent grades from 1 to 3 . These results suggest that the severity of $\mathrm{H}$. pylori infection of the stomach can be estimated by the UBT and that histopathologists might state whether the organism is present or absent, rather than making a quantitative statement as recommended in the Sydney system.
\end{abstract}

\section{Introduction}

The Sydney system for the classification of gastritis emphasises the importance of topographical, morphological and aetiological information. The system recommends that histopathologists make semiquantitative evaluations of gastritis, including the density of Helicobacter pylori in biopsy samples [1]. The density of $H$. pylori in such samples is correlated to the severity of gastritis $[2,3]$. The bacterial density of $H$. pylori in the stomach may be a predictive parameter for treatment success $[4,5]$, and may be associated with bacterial virulence factors in ulcer pathogenesis [6]. Biopsy samples can be examined by the rapid urease test, histological examination and conventional PCR, but these methods are not quantitative. Histological grading by the Sydney system can show the density of H. pylori in biopsy samples, but inter-observer

Received 20 Aug. 2001; revised version accepted 8 Oct. 2001.

Corresponding author: Dr Y. Eishi (e-mail: eishi.path@ tmd.ac.jp). differences are considerable [7]. More objective and quantitative methods are needed.

The urea breath test (UBT) is the most useful noninvasive test for $H$. pylori [8]. The bacterium produces a powerful urease, which is the basis of the UBT; labelled $\mathrm{CO}_{2}$ released by hydrolysis of labelled urea by urease into exhaled breath is measured. The density of $H$. pylori in gastric mucosa might be assessed indirectly by this quantitative method, but there is disagreement about whether correlation is significant $[9,10]$ or not $[11,12]$ between results of the UBT and the histologically estimated density of $H$. pylori in biopsy samples of the stomach.

This study used real-time (TaqMan) PCR to estimate numbers of $H$. pylori genomes in paraffin sections of biopsy samples. The specificity and sensitivity of this method to diagnose $H$. pylori infection were evaluated, as were those of other diagnostic methods, and the density of $H$. pylori genomes in biopsy samples was compared with the results of the UBT and the histological grading by the Sydney system. 


\section{Patients and methods}

\section{Patients}

Eighty-eight consecutive patients (mean age 50.7 SD 14.5 years; 60 men and 28 women; all Japanese) with dyspepsia attending this university hospital for diagnostic upper gastrointestinal endoscopy between Dec. 1997 and Jan. 2000 were enrolled. None of the patients had previously received antibiotic treatment for $H$. pylori, and all patients underwent a $\left[{ }^{13} \mathrm{C}\right]$ UBT just before the endoscopy. Gastrointestinal findings identified on the basis of endoscopy were recorded, and patients were classified into five groups according to the most severe findings: duodenal ulcer or ulcer scar $(\mathrm{n}=13)$, gastric ulcer or ulcer scar $(\mathrm{n}=27)$, erosions $(\mathrm{n}=6)$, gastritis $(\mathrm{n}=37)$ and normal $(\mathrm{n}=5)$. Duodenal and gastric ulcers were identified as circumscribed breaks in the mucosa that measured at least $5 \mathrm{~mm}$ across and surrounded a depressed area. Gastritis was classified as atrophic $(\mathrm{n}=28)$, superficial $(\mathrm{n}=5)$, hyperplastic $(\mathrm{n}=2)$, metaplastic $(\mathrm{n}=1)$ or verrucous $(\mathrm{n}=1)$.

\section{Entry procedure}

At entry, each patient underwent upper gastrointestinal endoscopy, histological evaluation of gastric mucosa and assessment of $H$. pylori status. Written informed consent to a biopsy of the gastric mucosa for diagnosis of $H$. pylori infection was obtained from each study participant in accordance with the Declaration of Helsinki (1964) and its later revisions.

\section{Diagnostic assays}

During endoscopy, three specimens each were obtained from the greater curvature of the antrum and from the middle portion of the greater curvature of the corpus. The first tissue sample was used for a CLO test (Delta West, Bentley, Western Australia). The test plate was brought to room temperature before use and monitored for colour change for $24 \mathrm{~h}$ after the addition of the gastric tissue. The second tissue sample was used for bacterial culture. Cultures for $H$. pylori were carried out on modified Skirrow agar with equine blood 10\% [13] incubated was for $5-7$ days at $37^{\circ} \mathrm{C}$ in a microaerobic atmosphere. H. pylori was identified by colony morphology and biochemical tests (urease, catalase and oxidase activities). The third tissue sample was used for histological examination and TaqMan PCR [14]. Tissues were placed in neutral buffered formalin $10 \%$ for $<24 \mathrm{~h}$ and embedded in paraffin wax. Tissue blocks were sliced $10 \mu \mathrm{m}$ thick and DNA of each biopsy specimen was extracted with a Dexpat kit (Takara Shuzo, Kyoto, Japan). Then 4- $\mu \mathrm{m}$ sections were stained with Giemsa and the colonisation of $H$. pylori on the gastric epithelium was graded on a 4-point scale of none (grade 0 ), mild (grade 1), moderate (grade 2) or severe (grade 3 ) according to the guidelines of the Sydney system [1]. Briefly, severe colonisation was defined as the presence of large groups of organisms on the surface and upper pits of two-thirds or more of the mucosal surface examined. Mild colonisation was defined as the presence of single organisms, or small groups, on less than one-third of the mucosal surface. Moderate colonisation was a condition between these two. Histological grading was made independently by two pathologists who were blinded to the results of other tests. The grading of 148 of the 176 samples $(84 \%)$ by the two pathologists was the same. For the samples with different results, consensus about the findings was reached by discussion with a third pathologist.

The UBT was done the day before the endoscopy. After an overnight fast, each patient provided a base-line breath sample. Patients swallowed $100 \mathrm{ml}$ of distilled water containing $100 \mathrm{mg}$ of $\left[{ }^{13} \mathrm{C}\right]$ urea. After $15 \mathrm{~min}$, a breath sample was collected in an air sample bag. The ${ }^{13} \mathrm{CO}_{2} /{ }^{12} \mathrm{CO}_{2}$ ratio was measured with a gas isotoperatio mass spectrometer (ABCA-NT; Europa Scientific, Crewe, UK). Results of the test were calculated as delta per mille change above the base line (before the administration of $\left[{ }^{13} \mathrm{C}\right]$ urea). Test results were considered positive if the ratio was $>5 \%$.

\section{Real-time (TaqMan) PCR}

TaqMan PCR was performed to amplify fragments of $16 \mathrm{~S}$ ribosomal RNA of $H$. pylori and of human $\beta$-globin DNA. For H. pylori, the primers used were HP-F, 5'-CTCATTGCGAAGGCGACCT-3' and HP-R, 5'-TCTAATCCTGTTTGCTCCCCA-3', and the TaqMan probe was HP-P, 5'-ATTACTGACGCTGATTGC GCGAAAGC-3'. For the human $\beta$-globin gene, primers were BG-F, 5'-TGCCTATCAGAAAGTGGT GGCT-3' and BG-R, 5'-GCTCAAGGCCCTTCATAA TATCC-3', and the TaqMan probe was BG-P, 5'-TGG CTAATGCCCTGGCCCACAA-3'. TaqMan PCR was performed in a reaction volume of $50 \mu$ l containing $5 \mu \mathrm{l}$ of sample DNA, $5 \mathrm{pmol}$ of each primer, 2 pmol of a TaqMan probe, $10 \mathrm{nmol}$ of each deoxynucleotide, $175 \mathrm{nmol}$ of $\mathrm{MgCl}_{2}, 1.25 \mathrm{U}$ of AmpliTaq Gold (PE Biosystems, Foster City, CA, USA) and 1X TaqMan buffer A (PE Biosystems). Amplification and detection were done with a detection system (GeneAmp 5700, PE Biosystems) with the profile of $95^{\circ} \mathrm{C}$ for $5 \mathrm{~min}$ and then 50 cycles of $95^{\circ} \mathrm{C}$ for $15 \mathrm{~s}$ and $60^{\circ} \mathrm{C}$ for $1 \mathrm{~min}$. The amount of bacterial and human DNA in samples was estimated from internal standard samples of serially diluted bacterial and human DNA, respectively. The amount of bacterial DNA was expressed in terms of the number of bacterial genomes, with $1.25 \times 10^{10} \mathrm{Da} /$ genome used in the conversion. The amount of human DNA in the same specimen was measured by TaqMan PCR of human $\beta$-globin DNA. The density of $H$. pylori in the biopsy samples was expressed as the numbers of bacterial genomes/pg of human DNA detected. 
The specificity of primers and probes for the DNAs of H. pylori and human $\beta$-globin was examined with the same procedures as for tissue samples. TaqMan analysis with each set of primers and probe for $H$. pylori or human $\beta$-globin DNA was done with $100 \mathrm{ng}$ of bacterial DNA from $H$. pylori (ATCC 43504D), Mycobacterium tuberculosis (RIMD 1337002), M. avium (IID 585), M. avium subsp. paratuberculosis (ATCC 19698), Bacteroides vulgatus (ATCC 8482), Escherichia coli (ATCC 11775), Propionibacterium acnes (ATCC 6919), P. granulosum (ATCC 25564) and Fusobacterium varium (JCM 3722), and of human DNA extracted from normal spleen tissue. TaqMan PCR specifically amplified the H. pylori DNA, and background values for DNA of other bacteria were less than one genome after PCR. TaqMan PCR of $\beta$-globin DNA specifically amplified human DNA, and bacterial genomes were not detected. Negative controls without bacterial DNA were included in every PCR; background values were always less than one genome. Samples with one or more bacterial genomes were counted as having positive results.

\section{Case definitions}

In comparisons of the five assays in this study, no single test was used as the criterion standard. A patient's $H$. pylori infection status was established by concordance of results of a minimum of two of the five tests. If the result for either of the two biopsy samples from the gastric antrum and corpus was positive, the patient was considered to have a positive result for that test.

\section{Statistical analysis}

Test-quality criteria (sensitivity, specificity, positive predictive value and negative predictive value) were compared in a four-field table. For comparison of the density of $H$. pylori genomes between different grades of the Sydney system, a non-parametric Kruskal-Wallis test and then a multiple comparison by Scheffe's F test were done. For evaluation of the correlation between the density of $H$. pylori genomes (mean of pairs of samples) and the results of UBTs of patients with and without $H$. pylori infection, Spearman's rank correla- tion coefficient was calculated. The agreement between observers was measured with the $\kappa$ statistic. $\kappa$ values $>0.75$ were taken to indicate a high level of agreement, those between 0.50 and 0.75 indicated moderate agreement, and those $<0.50$ indicated low agreement [15]. Differences with $p$ values $<0.05$ were considered to be significant.

\section{Results}

Results of the five tests were all negative for 15 patients and all positive for 62 patients, giving concordance in $77(88 \%)$ of the 88 patients. One patient each had one, two and three positive results, and eight patients had four positive results. The 72 patients $(82 \%)$ with two or more positive results were considered to be infected with $H$. pylori. The mean density of $H$. pylori genomes in pairs of biopsy samples from the gastric antrum and corpus of such patients ranged from 5 to 1125 ; the median was 168 (25th and 75th percentiles were 64 and 378, respectively).

Sensitivity and specificity in identification of the $H$. pylori status are shown in Table 1. TaqMan PCR had $100 \%$ sensitivity and specificity. The rapid urease test and culture both had $100 \%$ specificity but lower sensitivity. Histological examination and the UBT had sensitivity and specificity $<100 \%$. False negative results for $H$. pylori infection were found with the histological examination (one patient with a mean density of $36 \mathrm{H}$. pylori genomes), the rapid urease test (four patients with mean densities of 13, 17, 18 and 23), culture (two patients with mean densities of 538 and 1026) and the UBT (three patients with mean densities of 23, 62 and 114), but not with TaqMan PCR. False positive results for $H$. pylori infection were found with the histological examination (one patient with no $H$. pylori genomes detected) and the UBT (one patient with no such genomes detected), but not with the rapid urease test, culture or TaqMan PCR.

Fig. 1 shows the density of $H$. pylori genomes in tissue samples plotted against the histological grade by the Sydney system. Unexpectedly, H. pylori was detected

Table 1. Sensitivity, specificity, and positive and negative predictive values of five diagnostic tests for $H$. pylori infection in 88 patients undergoing gastrointestinal endoscopy

\begin{tabular}{lccrc}
\hline Test & $\begin{array}{c}\text { Sensitivity } \\
(\%)\end{array}$ & $\begin{array}{c}\text { Specificity } \\
(\%)\end{array}$ & $\begin{array}{c}\text { Positive PV } \\
(\%)\end{array}$ & $\begin{array}{c}\text { Negative PV } \\
(\%)\end{array}$ \\
\hline Invasive & & & & \\
$\quad$ TaqMan PCR & $100(72 / 72)^{*}$ & $100(16 / 16)$ & $100(72 / 72)$ & $100(16 / 16)$ \\
$\quad$ Histology & $99(71 / 72)$ & $94(15 / 16)$ & $99(71 / 72)$ & $94(15 / 16)$ \\
$\quad$ Rapid urease test & $94(68 / 72)$ & $100(16 / 16)$ & $100(72 / 72)$ & $80(16 / 20)$ \\
$\quad$ Culture & $97(70 / 72)$ & $100(16 / 16)$ & $100(72 / 72)$ & $89(16 / 18)$ \\
Non-invasive & $96(69 / 72)$ & $94(15 / 16)$ & $99(69 / 70)$ & $83(15 / 18)$ \\
$\quad$ Urea breath test & \multicolumn{3}{l}{} & \\
PV, predictive value. &
\end{tabular}




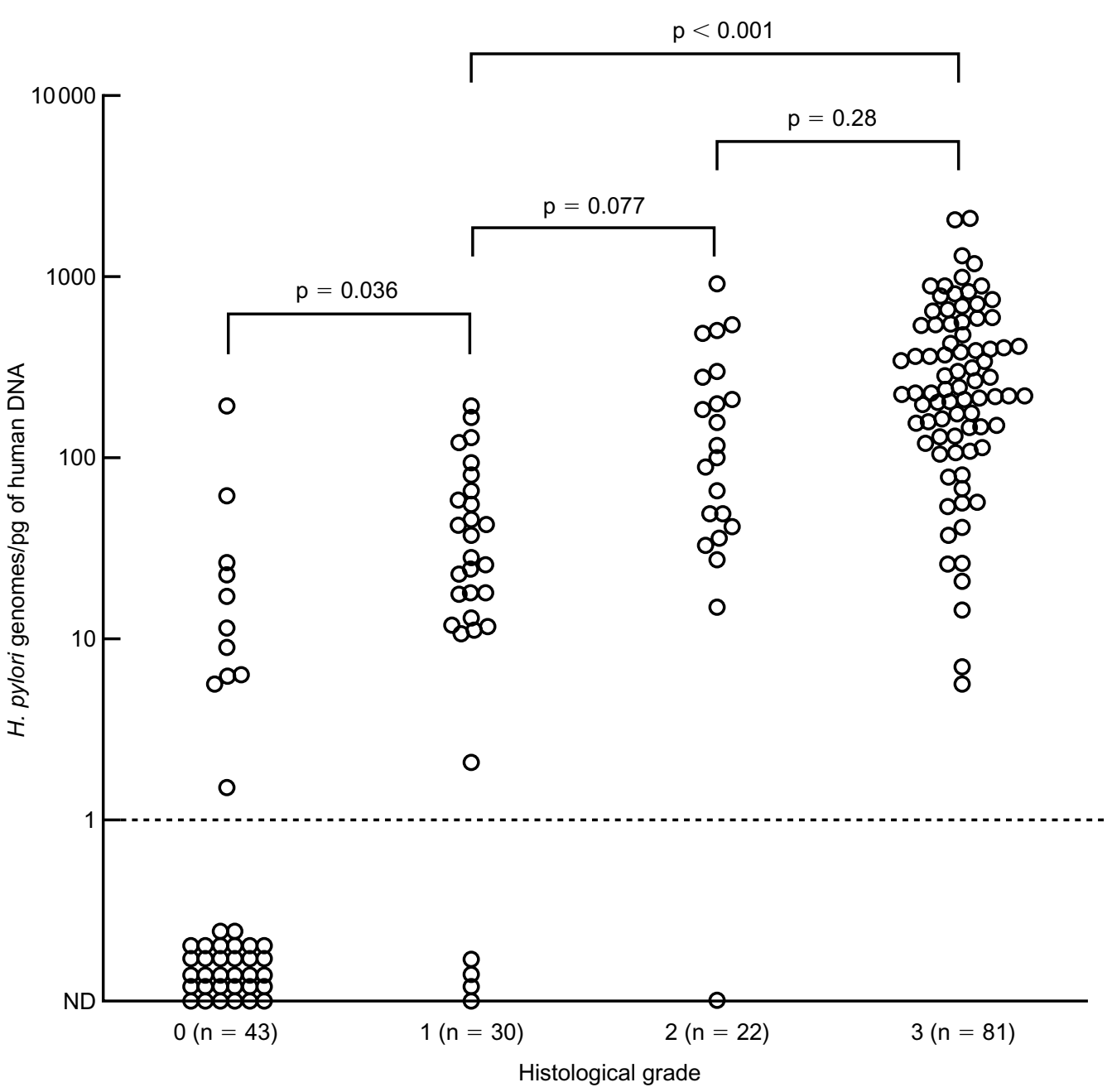

Fig. 1. Density of H. pylori genomes by quantitative PCR and histological grade by the Sydney system in 176 biopsy samples from the gastric antrum and corpus of 88 patients; ND, not detected.

in $11(26 \%)$ of the 43 samples graded 0 ; it was not detected in $4(13 \%)$ of the 30 samples graded 1 or in one $(5 \%)$ of the 22 samples graded 2. It was detected in all 81 samples graded 3. The mean density of $H$. pylori genomes was higher in grade $1(\mathrm{p}=0.036)$, grade $2(p<0.001)$ and grade $3(p<0.001)$ than in grade 0 , with no significant differences between adjacent grades from grades 1 to 3 . Grades 1 and 3 were significantly different $(\mathrm{p}<0.001)$.

Fig. 2 shows the mean density of $H$. pylori genomes in paired biopsy samples from the gastric antrum and corpus of individual patients plotted against the UBT values for the 88 patients. Correlation between the two was significant $(r=0.81, \mathrm{p}<0.001)$. When the 16 patients without $H$. pylori infection were excluded from the analysis, correlation was still significant $(r=0.67, \mathrm{p}<0.001)$.

\section{Discussion}

TaqMan PCR has been used for quantitative estimates of the numbers of bacteria such as M. tuberculosis
[14], Listeria monocytogenes [16], Salmonella spp. [17] and Yersinia pestis [18]. However, to our knowledge, the technique has not been used for such estimates of $H$. pylori. Assays based on conventional PCR to detect the presence of $H$. pylori DNA with different gene targets have been described [19-23]. Among them, Weiss et al. [23] used conventional PCR to detect the presence of a 136-bp fragment of $H$. pylori 16S rRNA in formalin-fixed paraffin-embedded gastric biopsy specimens from 95 patients with dyspepsia and found 94\% sensitivity and 100\% specificity. The present study used TaqMan PCR to amplify an 85-bp fragment of $H$. pylori $16 \mathrm{~S}$ rRNA and found $100 \%$ sensitivity and $100 \%$ specificity. The sensitivity may be greater partly because the amplicon was shorter, but a more important factor may be other differences in the detection procedure itself. The analytical sensitivity of TaqMan PCR is close to that of the nested PCR; both methods can detect as few as 10 copies of a standard plasmid [24]. In addition to its quantitative results, advantages of TaqMan PCR compared with conventional nested PCR are shorter working time (shorter cycling time and no gel electrophoresis), larger numbers of samples (up to 96) 


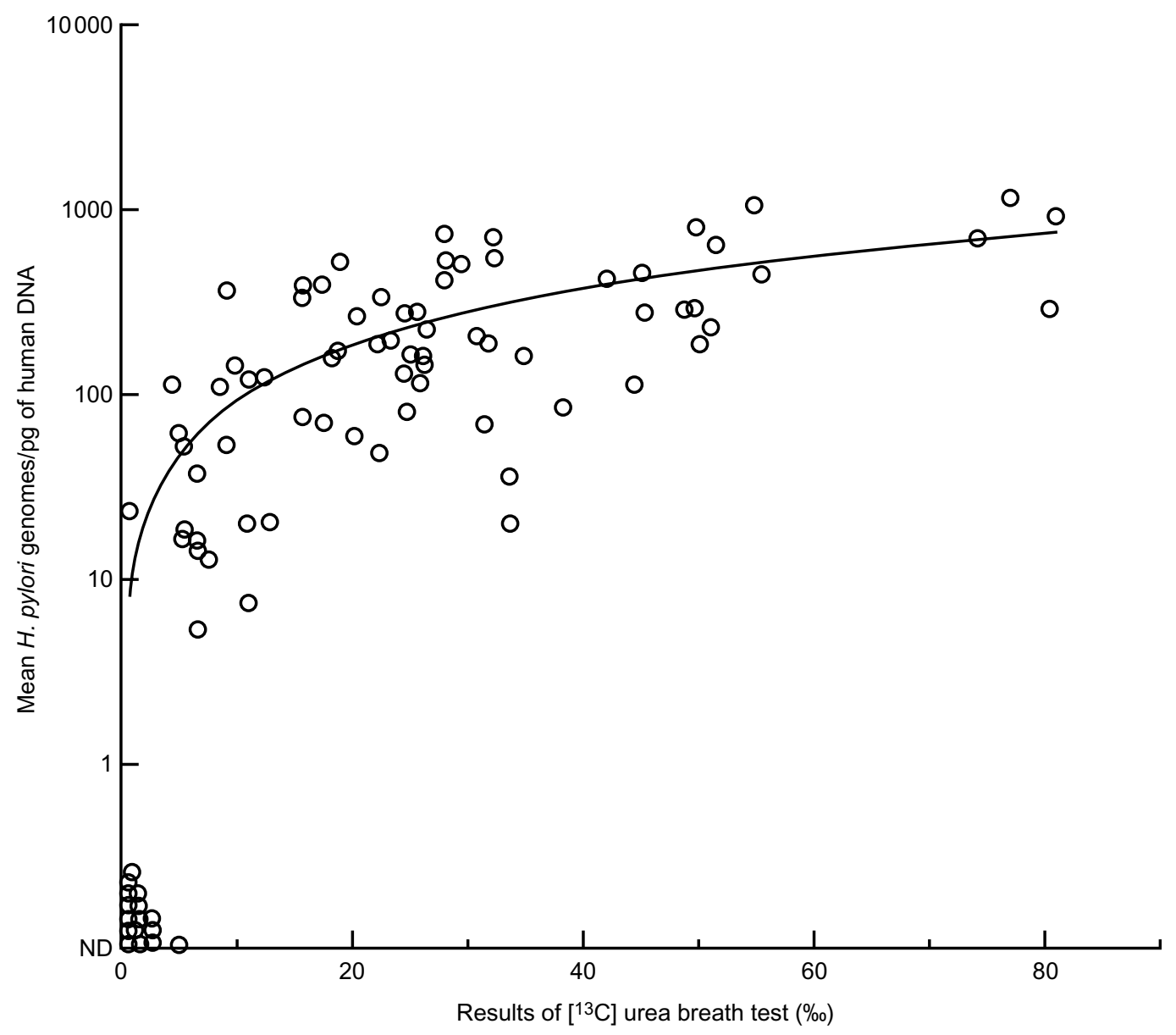

Fig. 2. Mean density of $H$. pylori genomes in paired biopsy samples from the gastric antrum and corpus of 88 patients and results of the $\left[{ }^{13} \mathrm{C}\right]$ urea breath test. ND, not detected; $r=0.81 ; \mathrm{p}<0.001$.

that can be worked with in a single round, and lower risk of contamination because amplified samples are not further handled.

For accurate evaluation of $H$. pylori density in gastric mucosa, the density of $H$. pylori in the biopsy samples was expressed as the number of bacterial genomes/pg of human DNA found by PCR of $\beta$-globin DNA. Such correction may be useful when specimens differ in size, including when histological sections differ in thickness. Furthermore, correction may be needed to prevent false-negative results and to adjust results affected by fixation of the tissues with formalin. PCR amplification may be suppressed by a specific inhibitor in the sample DNA solution, preventing positive findings. With the method used in the present study, when amplification is suppressed by a specific inhibitor, not only $H$. pylori DNA but also human $\beta$-globin DNA is undetectable. In this way, true negative results can be distinguished from false negative results. DNA in paraffin samples degenerates when the samples are fixed with formalin. A fixation time of $<24 \mathrm{~h}$ and target fragment sizes of $85-120$ bp were chosen on the basis of studies of the relationship between fixation time and the amplifiable fragment size [25]. Not only samples prepared especially for this study but also routine samples from other laboratories that have been processed differently may be examined accurately by the method reported here, because H. pylori and human DNA in a sample probably are denatured at a similar rate during preparation.

In this study, false negative results from histological examinations, the rapid urease test and the UBT were considered to indicate the lower sensitivity of these methods, because the density of $H$. pylori genomes in most of the patients with such false negative results was lower than the 25th percentile for patients with true positive results. The values for the density of $H$. pylori in the two false negative results by culture suggest that the problem was not sensitivity but probably a technical failure in the cultivation of $H$. pylori ( $H$. pylori is often difficult to isolate in culture). False positive results by histological examination and the UBT may have been caused by non-specific bacterial staining with Giemsa or mistakes made during the UBT. Of the variety of diagnostic methods tried, the real-time quantitative PCR reported here had the highest sensitivity and specificity in the evaluation of $H$. pylori infection in untreated patients with dyspepsia.

TaqMan PCR detected H. pylori genomes in 11 of the 43 samples with negative results by histological 
examination. All these samples were from patients infected with $H$. pylori according to the study criteria. It was likely that histological results for those samples were false negatives. The four PCR-negative samples of grade 1 and the one such sample of grade 2 may have given false positive results.

The reliability of the histological grading of $H$. pylori density in this study was similar to that reported by others. Two of the authors examined two biopsy samples (from the gastric antrum and corpus), stained with Giemsa, from 88 patients (total, 176 samples) and graded the H. pylori density. Results agreed for 65 of the antral biopsies $(74 \% ; \kappa=0.62)$ and for 79 of the corporal biopsies $(90 \% ; \kappa=0.84)$; the $\kappa$ values were close to those $(0.70-0.89)$ reported by Andrew et al. [26].

The method reported here made possible objective estimation of bacterial density in the gastric mucosa. Moshkowitz et al. [4] reported that bacterial density, as assessed by urease activity, can help to predict $H$. pylori eradication. They suggested that the UBT done before treatment can be used to identify patients for whom the standard therapeutic regimen should be modified. Sheu et al. [5] suggested that histologically estimated bacterial density in gastric mucosa also can be used to predict $H$. pylori eradication in patients with duodenal ulcer bleeding. Atherton et al. [6] reported that quantitative culture gave greater reproducibility of results than histological examination, and that $H$. pylori density in vivo is associated with the $\operatorname{cag} A$ and vacA genotypes (bacterial virulence determinants), the extent of gastric inflammation, and whether there is duodenal ulceration. Thus, measurement of $H$. pylori density in gastric mucosa may be useful in studies of whether the severity of the infection of gastric mucosa has clinical or pathological meaning. However, the semiquantitative histological examination of the Sydney system cannot be used for this purpose, because of the lack of a significant difference between adjacent histological grades 1-3. It might be appropriate for histopathologists to conclude whether the organism is present or absent, rather than making a quantitative estimate as recommended in the Sydney classification.

The numerical results of the UBT are a function of total urease activity within the stomach, so the UBT might be a quantitative index of the density of gastric H. pylori colonisation. There have been many studies reporting inconsistent results about the relationship between results of the UBT and those of histologybased semiquantitative measures of bacterial infection. Such inconsistency may have arisen from inter-observer differences [7] or inaccuracy in the estimation of bacterial density by a subjective histology-based method. Epple et al. [27] found significant correlation $(r=0.78)$ in results by these two methods, but they reported that the correlation was high only when many patients with histologically negative results (there were
49 in the 126 patients) were included in the analysis; when excluded, correlation was weak $(r=0.33)$. They suggested that the weakness of correlation when $H$. pylori-negative patients were excluded was due to overlapping of values obtained by the UBT in the three histologically defined groups with low, moderate and high colonisation densities, and concluded that the UBT is not suitable for estimation of the density of $H$. pylori colonisation.

In the present study, objective and numerical results of the $H$. pylori density in the stomach were compared with the results of UBT, and significant correlation was found between the two methods both with and without results of $H$. pylori-negative patients. Differences in $H$. pylori strains may account for differences in urease activity [28], so quantitative results of the UBT may be inaccurate. Furthermore, the corpus and fundus of the stomach may be coated unevenly with urea if the protocol of the UBT, including positioning of the patient, is not strictly followed. The mean density of $H$. pylori genomes in paired biopsy samples from the gastric antrum and corpus may not reflect total bacterial numbers of $H$. pylori in the stomach. Even with these possible drawbacks, correlation was significant in this study. Information from the UBT is useful not only in identifying $H$. pylori infection but also in indirect assessment of the numbers of $H$. pylori in the stomach.

This study was financed in part by grants from the Ministry of Health and Welfare, Japan.

\section{References}

1. Price AB. The Sydney system: histological division. J Gastroenterol Hepatol 1991; 6: 209-222.

2. Satoh K, Kimura K, Yoshida Y, Kasano T, Kihira K, Taniguchi Y. A topographical relationship between Helicobacter pylori and gastritis: quantitative assessment of Helicobacter pylori in the gastric mucosa. Am J Gastroenterol 1991; 86: 285-291.

3. Loffeld RJLF, Potters HVPJ, Arends JW, Stobberingh E, Flendrig JA, van Spreeuwel JP. Campylobacter associated gastritis in patients with non-ulcer dyspepsia. J Clin Pathol 1988; 41: 85-88.

4. Moshkowitz M, Konikoff FM, Peled Y et al. High Helicobacter pylori numbers are associated with low eradication rate after triple therapy. Gut 1995; 36: 845-847.

5. Sheu B-S, Yang H-B, Su I-J, Shiesh S-C, Chi C-H, Lin X-Z. Bacterial density of Helicobacter pylori predicts the success of triple therapy in bleeding duodenal ulcer. Gastrointest Endosc 1996; 44: 683-688.

6. Atherton JC, Tham KT, Peek RM, Cover TL, Blaser MJ. Density of Helicobacter pylori infection in vivo as assessed by quantitative culture and histology. $J$ Infect Dis 1996; 174: 552-556.

7. el-Zimaity HMT, Graham DY, al-Assi MT et al. Interobserver variation in the histopathological assessment of Helicobacter pylori gastritis. Hum Pathol 1996; 27: 35-41.

8. Atherton JC, Spiller RC. The urea breath test for Helicobacter pylori. Gut 1994; 35: 723-725.

9. Sheu B-S, Lee S-C, Yang H-B, Lin X-Z. Quantitative result of ${ }^{13} \mathrm{C}$ urea breath test at 15 minutes may correlate with the bacterial density of $\mathrm{H}$. pylori in the stomach. Hepatogastroenterology 1999; 46: 2057-2062.

10. Ellenrieder V, Glasbrenner B, Stoffels C et al. Qualitative and semi-quantitative value of a modified ${ }^{13} \mathrm{C}$-urea breath test for identification of Helicobacter pylori infection. Eur J Gastroenterol Hepatol 1997; 9: 1085-1089. 
11. Hilker E, Domschke W, Stoll R. ${ }^{13}$ C-urea breath test for detection of Helicobacter pylori and its correlation with endoscopic and histologic findings. $J$ Physiol Pharmacol 1996; 47: 79-90.

12. Logan RPH, Polson RJ, Misiewicz JJ et al. Simplified single sample ${ }^{13}$ carbon urea breath test for Helicobacter pylori: comparison with histology, culture, and ELISA serology. Gut 1991; 32: $1461-1464$.

13. Dent JC, McNulty CAM. Evaluation of a new selective medium for Campylobacter pylori. Eur J Clin Microbiol Infect Dis 1988; 7: 555-558.

14. Desjardin LE, Chen Y, Perkins MD, Teixeria L, Cave MD, Eisenach KD. Comparison of the ABI 7700 system (TaqMan) and competitive PCR for quantification of IS6110 DNA in sputum during treatment of tuberculosis. J Clin Microbiol 1998; 36: 1964-1968.

15. Svanholm H, Starklint H, Gundersen HJG, Fabricius J, Barlebo $\mathrm{H}$, Olsen S. Reproducibility of histomorphologic diagnoses with special reference to the kappa statistic. APMIS 1989; 97: 689-698.

16. Bassler HA, Flood SJA, Livak KJ, Marmaro J, Knorr R, Batt CA. Use of a fluorogenic probe in a PCR-based assay for the detection of Listeria monocytogenes. Appl Environ Microbiol 1995; 61: 3724-3728.

17. Chen S, Yee A, Griffiths $\mathrm{M}$ et al. The evaluation of a fluorogenic polymerase chain reaction assay for the detection of Salmonella species in food commodities. Int $J$ Food Microbiol 1997; 35: 239-250.

18. Higgins JA, Ezzell J, Hinnebusch BJ, Shipley M, Henchal EA, Ibrahim MS. 5' nuclease PCR assay to detect Yersinia pestis. J Clin Microbiol 1998; 36: 2284-2288.

19. Clayton CL, Kleanthous H, Coates PJ, Morgan DD, Tabaqchali S. Sensitive detection of Helicobacter pylori by using polymerase chain reaction. J Clin Microbiol 1992; 30:
192-200.

20. Engstrand L, Nguyen A-MH, Graham DY, el-Zaatari FAK Reverse transcription and polymerase chain reaction amplification of rRNA for detection of Helicobacter species. J Clin Microbiol 1992; 30: 2295-2301.

21. Hammar M, Tyszkiewicz T, Wadström T, O’Toole PW. Rapid detection of Helicobacter pylori in gastric biopsy material by polymerase chain reaction. J Clin Microbiol 1992; 30: 54-58.

22. Wang JT, Lin JT, Sheu JC, Yang JC, Chen DS, Wang TH. Detection of Helicobacter pylori in gastric biopsy tissue by polymerase chain reaction. Eur J Clin Microbiol Infect Dis 1993; 12: $367-371$

23. Weiss J, Mecca J, da Silva E, Gassner D. Comparison of PCR and other diagnostic techniques for detection of Helicobacter pylori infection in dyspeptic patients. J Clin Microbiol 1994; 32: $1663-1668$.

24. Pusterla N, Huder JB, Leutenegger CM, Braun U, Madigan JE, Lutz H. Quantitative real-time PCR for detection of members of the Ehrlichia phagocytophila genogroup in host animals and Ixodes ricinus ticks. J Clin Microbiol 1999; 37: 1329-1331.

25. Greer CE, Lund JK, Manos MM. PCR amplification from paraffin-embedded tissues: recommendations on fixatives for long-term storage and prospective studies. PCR Methods Appl 1991; 1: 46-50.

26. Andrew A, Wyatt JI, Dixon MF. Observer variation in the assessment of chronic gastritis according to the Sydney system. Histopathology 1994; 25: 317-322.

27. Epple HJ, Kirstein FW, Bojarski C et al. ${ }^{13} \mathrm{C}$-urea breath test in Helicobacter pylori diagnosis and eradication. Correlation to histology, origin of 'false' results, and influence of food intake. Scand J Gastroenterol 1997; 32: 308-314.

28. Mobley HL, Cortesia MJ, Rosenthal LE, Jones BD. Characterization of urease from Campylobacter pylori. J Clin Microbiol 1988; 26: $831-836$. 